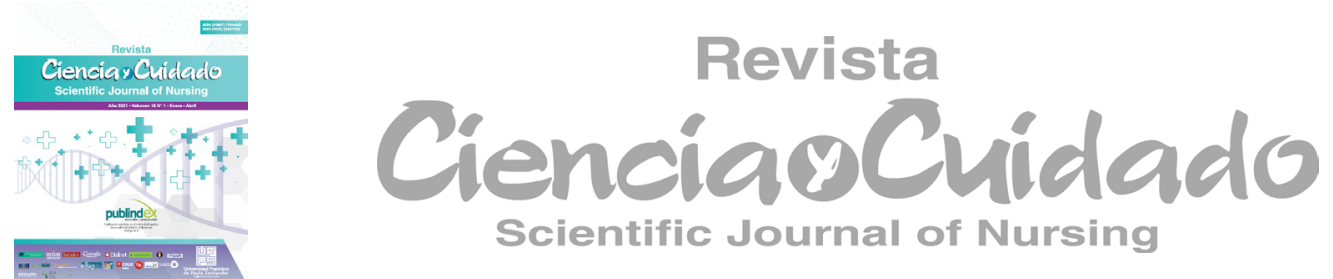

Articulo Original

\title{
Significados del cuidado construidos por cuidadores de personas mayores ${ }^{\dagger}$
}

\author{
Significance of caring built by caretakers of elderly people.
}

\section{Significado do cuidado construido por cuidadores de pessoas idosas.}

\author{
María Isabel Zuluaga-Callejas ${ }^{1}$ \\ María Eumelia Galeano-Marin ${ }^{2}$ \\ Caterin Bibiana Giraldo-Giraldo ${ }^{3}$ \\ Viviana Marcela Vélez-Escobar ${ }^{4}$ \\ Sandra Milena Sánchez-Vallejo \\ Alex Mauricio Lopera-Arango ${ }^{6}$ \\ Juliana Ortiz-Berrio ${ }^{7}$ \\ Dagoberto Barrera-Valencia ${ }^{8}$
}

\section{Resumen}

Introducción: En el marco de un proceso formativo en 5 ciudades de Colombia, impartido por la Universidad de Antioquia y financiado por PROTECCIÓN S.A. entre los años 2018 - 2020, se planteó como objetivo: analizar los significados del cuidado que construyen los cuidadores de personas mayores en las ciudades de Rionegro, Bogotá, Cali, Pereira y Medellín. Materiales y métodos: 202 participantes, con edades comprendidas entre los 21 y los 73 años. Se desarrolló un estudio etnográfico particularista y colaborativo. Se realizaron 6 entrevistas individuales a cuidadores y expertos; se desarrolló observación participante y no participante de las clases del diplomado y de algunos contextos de cuidado. Resultados: El amor, la preservación de la existencia, el servicio, el sentido de vida y la responsabilidad fueron los significados que sobresalieron en los cuidadores de personas mayores, la transformación de estos significados se denota en la dedicación que destinan para la persona mayor cuidada, permitiendo incluso, una perspectiva de autocuidado. Conclusiones: La labor de cuidar es un acto que compromete la vida emocional tanto del cuidador como de la persona mayor cuidada. Se identificó que el proceso de formación favoreció la creación de redes de cuidadores, los cuáles en la medida del proceso formativo, evidenciaron capacidad de transformar visiones y significados; replantear situaciones particulares; comprender la necesidad de cuidar de sí, para cuidar a otro; la creación de lazos de solidaridad; las experiencias del trabajo interdisciplinar y el interés por capacitarse y compartir conocimientos.

Palabras claves: Cuidado de personas mayores, envejecimiento, vejez, significados del cuidado, personas mayores, cuidadores.
Autor de correspondencia*

1*Psicóloga. isabel.zuluaga@udea.edu.co (ID

${ }^{2}$ Socióloga.galeanomarin@gmail.com (iD

3 Estudiante de psicología. giraldogiraldo96@gmail.com.

${ }^{4}$ Psicóloga. velez61@gmail.com. (D)

5 Gerontóloga. sandramsanchez0930@, gmail.com.

${ }^{6}$ Enfermero. alex.lopera@udea.edu.co. (iD

${ }^{7}$ Antropóloga. juli.ortizberri@gmail.com.

8 Psicólogo. dagoberto.barrera@udea.edu. co. (iD)

Recibido: 1 octubre 2020

Aprobado: 16 diciembre 2020

Para citar este articulo: Zuluaga-Callejas MI, Galeano-Marín ME, Giraldo-Giraldo CB, Vélez-Escobar VM, Sánchez-Vallejo SM, Lopera-Arango AM, et al. Significados del cuidado construidos por cuidadores de personas mayores. Rev. cienc. cuidad. 2021;18(1):54-65. https://doi. org/10.22463/17949831.2741

(C) Esta obra está bajo licencia internacional CC-BY-NC-ND (C) $\Theta \Theta$ 


\begin{abstract}
Introduction: Within the framework of a training process in 5 cities of Colombia, given by the University of Antioquia and financed by PROTECCIÓN S.A. between the years 2018 - 2020, the objective was set: to analyze the meanings of the care that the caregivers of the elderly build in the cities of Rionegro, Bogotá, Cali, Pereira and Medellín. Materials and methods: 202 participants, between 21 and 73 years old. A particularist and collaborative ethnographic study was developed. Six individual interviews were conducted with caregivers and experts; participant and non-participant observation of the diploma classes and some care contexts was developed. Results: love, preservation of existence, service, meaning of life and responsibility were the meanings that stood out in the caregivers of older people, the transformation of these meanings is denoted in the dedication they give to the older person being cared for, even allowing a perspective of self-care. Conclusions: the work of caring is an act that compromises the emotional life of both the caregiver and the elderly carer. It was identified that the formation process favored the creation of caregivers' networks, which in the measure of the formation process, showed capacity to transform visions and meanings; to rethink particular situations; to understand the need to take care of oneself, to take care of another; the creation of solidarity ties; the experiences of interdisciplinary work and the interest in training and sharing knowledge.
\end{abstract}

Key words: care of the elderly, aging, old age, meanings of care, elderly, caregivers.

\title{
Resumo
}

Introdução: No marco do processo formativo em cinco cidades da Colômbia, oferecido pela Universidade de Antioquia e financiado por PROTECCIÓN S.A entre os anos 2018 e 2020 planteou-se o objetivo de analisar os significados de cuidado construídos por cuidadores de pessoas idosas nas cidades de Rionegro, Bogotá, Cali, Pereira e Medellín. Materiais e métodos: 202 participantes, com idades entre 21 e 73 anos. Desenvolveu-se um estudo etnográfico particularista e colaborativo, realizaram-se 6 entrevistas individuais a cuidadores e expertos; desenvolveu-se observação participante e não participante das aulas do curso e de alguns contextos de cuidado. Resultados: O amor, a preservação da existência, o serviço, o sentido da vida e a responsabilidade foram os significados que se destacaram nos cuidadores de pessoas idosas. A transformação desses significados evidenciou-se na dedicação à pessoa idosa, permitindo incluso a perspectiva do autocuidado. Conclusões: O papel de cuidar é um ato que compromete a vida emocional tanto do cuidador como da pessoa idosa cuidada, identificou-se que o processo de formação favorecei a criação de redes de cuidadores, os quais na medida do processo formativo, evidenciaram capacidade de transformar visões e significados; reformular situações particulares; compreender a necessidade de cuidar de si para cuidar ao outro; a criação de alianças de solidariedade; as experiências do trabalho interdisciplinar e o interesse por capacitar-se e compartir conhecimentos.

Palavras-chave: Assistência do adulto, envelhecimento, cuidado, idoso, cuidadores.

\section{Introducción}

El envejecimiento es un proceso natural y transversal del curso de la vida. Se habla de envejecimiento poblacional cuando hay un aumento de la proporción de personas mayores con respecto al total de la población (1), a consecuencia de dos fenómenos: la transición demográfica y la transición epidemiológica. La vejez, a diferencia del envejecimiento, se entiende como "la última etapa del curso de vida en la que confluyen factores individuales, colectivos y contextuales como efecto de elecciones individuales y el tránsito de distintas condiciones de vida" (1) lo que indica que la vejez es un momento específico y focalizado, permeado por una construcción social, económica, política y cultural (2).

Colombia es un país con una transición demográfica moderada, representada en el incremento de la esperanza de vida y la disminución de la fecundidad (1). 
María Isabel Zuluaga-Callejas • María Eumelia Galeano-Marín • Caterin Bibiana Giraldo-Giraldo • Viviana Marcela Vélez-Escobar - Sandra Milena Sánchez-Vallejo • Alex Mauricio Lopera-Arango • Juliana Ortiz-Berrio • Dagoberto Barrera -Valencia

El 10.5\% de la población es mayor de 60 años. Sumado a esto, el envejecimiento se ha dado en medio de un contexto de pobreza aguda, desigualdad del ingreso y persistente inequidad social (3), factores que repercuten directamente en la calidad de vida de las personas mayores y de sus cuidadores, quienes en su mayoría son mujeres.

A partir de la Ley 1413 de 2010 (4) se regula la inclusión de la economía del cuidado en el sistema de cuentas nacionales, con el objeto de medir la contribución de la mujer en el desarrollo económico y social del país, por un lado, y como herramienta fundamental para la definición e implementación de políticas públicas, por el otro. Asimismo, el Plan Nacional de Desarrollo 2014-2018 (5) creó las bases institucionales, técnicas y económicas para desarrollar el Sistema Nacional de Cuidados. Sin embargo, el país aún va a paso lento frente a la regulación y protección social integral de los cuidadores de personas mayores, ya que la normativa no se refleja en decretos ni leyes que favorezcan la labor de cuidar.

En la encuesta SABE Colombia (6), sobre la situación de salud, bienestar y envejecimiento en Colombia, se informa que de los cuidadores familiares, el 16.7\% son personas mayores de 60 años que cuidan a otras personas mayores; $83.9 \%$ son mujeres, $84.9 \%$ no cotizan a fondos de pensión y únicamente el $7 \%$ tienen remuneración. Además, quienes cuidan a las personas mayores están dedicadas en gran parte a las tareas y oficios del hogar, con una escasa protección socioeconómica, en su mayoría son hijas o cónyuge de la persona que cuidan. Dedican 7 días a la semana y conviven con la persona cuidada, sin los recursos ni la formación suficiente para cumplir con su labor de cuidado; también reportan cansancio y síntomas ligados a ésta, sumado al poco apoyo por parte del sistema de salud o del Estado. Por su parte, los cuidadores formales en ámbitos institucionales, son en su mayoría mujeres entre los 20 a 39 años, prima el estado civil soltero (a), con largas jornadas laborales, alto nivel de estrés y una desproporción entre las demandas y los recursos laborales.

Adicional a estas condiciones, están los diversos problemas que padecen los cuidadores familiares de una persona con enfermedad o con discapacidad, puesto que el cuidador sufre cambios en todas las áreas de desempeño, como son su salud física y psicológica; igualmente, pasa por diversas emociones que le impiden mantener una sensación de bienestar. Estos problemas también se presentan en las personas que laboran en los servicios de salud y programas para la atención de las personas mayores (7). Por esto, es menester atender el envejecimiento con una lógica que integre políticas públicas, sociales y de salud, con un enfoque de curso de vida, además del diseño e implementación de programas y servicios estatales que ofrece el Estado y los distintos organismos que se ven convocados a trabajar por el envejecimiento y por sus cuidadores.

De La Cuesta (8) indicó que el sistema de cuidado, especialmente el informal, requiere de una serie de apoyos técnicos, económicos y emocionales que fortalezcan la labor. Cuando en una familia se cuida a un miembro con dependencia funcional y decide asumir el cuidado informal, se alteran los roles de sus miembros, particularmente de aquel que asume el papel de cuidador principal, quien puede verse afectado en su salud y de forma especial, en su salud mental (9). Esta situación se puede agravar si además no se cuenta con factores protectores que le sirvan de soporte.

De esta manera, afrontar el cuidado de una persona mayor incrementa las necesidades de apoyo médico, institucional y familiar (10). Los cuidadores pueden presentar sobrecarga física y emocional que fácilmente deriva en «el síndrome del cuidador», con el riesgo de que se convierta en un enfermo secundario (11). Este panorama crea retos para la salud pública lo que debe motivarla a fomentar nuevas iniciativas para promover la vida digna de la población que envejece (7). En este sentido, se debe precisar que los "aspectos relacionados con la salud mental, la discapacidad funcional y el sentirse maltratado por parte de los familiares reduce la calidad de vida" (12-1). Así entonces, uno de los principales retos para afrontar el envejecimiento está relacionado con la necesidad de cuidado y de cuidadores dispuestos a brindar el acompañamiento que requieren los mayores, especialmente cuando pierden su capacidad funcional y pasan a ser dependientes en el desarrollo de las actividades básicas diarias.

Conviene subrayar que esta situación constituye un tema sensible de intervención e investigación, pues supone el despliegue de recursos económicos, sociales, familiares y de servicios, que requiere de planeación y la concertación. En esa ruta, se realizó esta investigación, en el marco del desarrollo de un diplomado, con el ánimo de generar líneas de profundización e intervención que mejoren la calidad de vida del cuidador y de las personas mayores en Colombia, reconociendo la diversidad de contextos.

Se debe mencionar, además que los significados son el 
resultado de una construcción, que emerge mediante la relación con los otros y que permite la modificación de las conductas para facilitar ese encuentro. En consecuencia, este trabajo cobra sentido por la escasez de estudios focalizados en los cuidadores de personas mayores y en lo que dicha experiencia significa para ellos

\section{Objetivos}

\section{Objetivo general}

Analizar los significados del cuidado que construyen los cuidadores de personas mayores, de acuerdo con la situación de cuidado de los participantes de un proceso de formación en Rionegro, Bogotá, Cali, Pereira y Medellín.

\section{Objetivos Específicos}

- Identificar la situación de cuidado de acuerdo con las condiciones económicas, sociales, familiares y la carga de cuidado, en relación a los efectos, los requisitos y las expresiones del cuidado.

- Contrastar los significados del cuidado identificados al inicio y al final del proceso formativo.

- Plantear las dimensiones del cuidado más significativas en las situaciones estudiadas.

\section{Materiales y Métodos}

El enfoque cualitativo que se asume en esta investigación hace referencia a los rasgos característicos sobre el interés por el significado, la interpretación y el énfasis respecto a la importancia del contexto y de los procesos en que participan los sujetos de este estudio, permitiendo la construcción de relaciones intersubjetivas basadas en el diálogo y la reflexión, entre el equipo de investigación y los cuidadores de personas mayores, para comprender estos significados. Este estudio se basó, por tanto, en el análisis subjetivo a partir de la interacción con los participantes, lo que permitió hacer una interpretación referida a los contextos y situaciones en los que se encuentran inmersos los cuidadores y de los significados que construyen en el proceso de cuidar a personas mayores. Esta indagación se realizó en Rionegro, Medellín, Cali, Pereira y Bogotá, ciudades ubicadas en regiones sociodemográficas con características particulares. Por tanto, esta investigación se centra en los cui- dadores que, como sujetos, en situaciones específicas, día a día viven su cotidianidad en interacción con otros individuos (personas mayores que cuidan, familiares, vecinos, instituciones) y en esa interrelación construyen significados sobre el cuidar, sobre sí mismos y sobre su contacto con el entorno inmediato.

Así pues, teniendo en cuenta el incremento en el índice de envejecimiento en distintas regiones de Colombia, se eligieron las ciudades de Rionegro, Medellín, Cali, Pereira y Bogotá. Participaron 202 individuos, con edades comprendidas entre los 21 y los 73 años, mayormente mujeres. La participación en el diplomado y en la investigación fue voluntaria y se orientó bajo el enfoque cualitativo mediante un estudio etnográfico particularista, centrado en la situación y contexto de cuidado, y a la etnografía colaborativa. A todos los participantes se les solicitó diligenciar dos encuestas, una al inicio y otra al final del diplomado, con las preguntas: ¿qué significa cuidar?, ¿Por qué?, ¿Para qué? Se hizo observación participante de las sesiones del diplomado y algunos contextos de cuidado familiar e institucional a partir de visitas en hogares e instituciones. Para controlar los sesgos en la recolección y análisis de la información, se recurrió a entrevistas realizadas a cuidadores a quienes se les nombró como expertos en relación a sus experiencias en diferentes situaciones de cuidado. Además se acudió al diálogo con otros investigadores del grupo que trabajan el tema y, finalmente, se socializaron los resultados con los participantes en cada uno de los municipios donde se desarrolló el diplomado. Los datos se analizaron en el Software Atlas ti versión 7.5.4.

En los aspectos éticos se asumieron como orientaciones básicas: contar con el consentimiento informado de las instituciones y de los cuidadores; guardar la confidencialidad y el anonimato de los participantes a excepción de que alguno manifestase expresamente su deseo de ser nombrado; la integralidad en el proceso investigativo manteniendo la fidelidad de los datos, la contrastación, la validación de la información y la exactitud en el uso de fuentes. Por último, se socializaron los resultados de la investigación mediante la discusión de los hallazgos tanto con la institución que financia la investigación como con los participantes.

\section{Resultados}

\section{Situación y contexto de cuidado}

En la figura 1 se muestran las situaciones psicológicas, sociales y económicas que viven a diario los que 
María Isabel Zuluaga-Callejas • María Eumelia Galeano-Marín • Caterin Bibiana Giraldo-Giraldo • Viviana Marcela Vélez-Escobar - Sandra Milena Sánchez-Vallejo • Alex Mauricio Lopera-Arango • Juliana Ortiz-Berrio • Dagoberto Barrera -Valencia

se desempañan como cuidadores de personas mayores, donde se observa que su labor está rodeada de escasos recursos económicos para su sostenimiento, ausencia de espacios adecuados que favorezcan la ayuda y el libre desplazamiento de la persona cuidada. Se evidencia también la presencia de personas mayores que son cuidadas por personas de su misma edad y a largo plazo, causando implicaciones fisiológicas en los cuidadores como consecuencia del cuidado, por ejemplo, el desgaste emocional y el insomnio, entre otros.

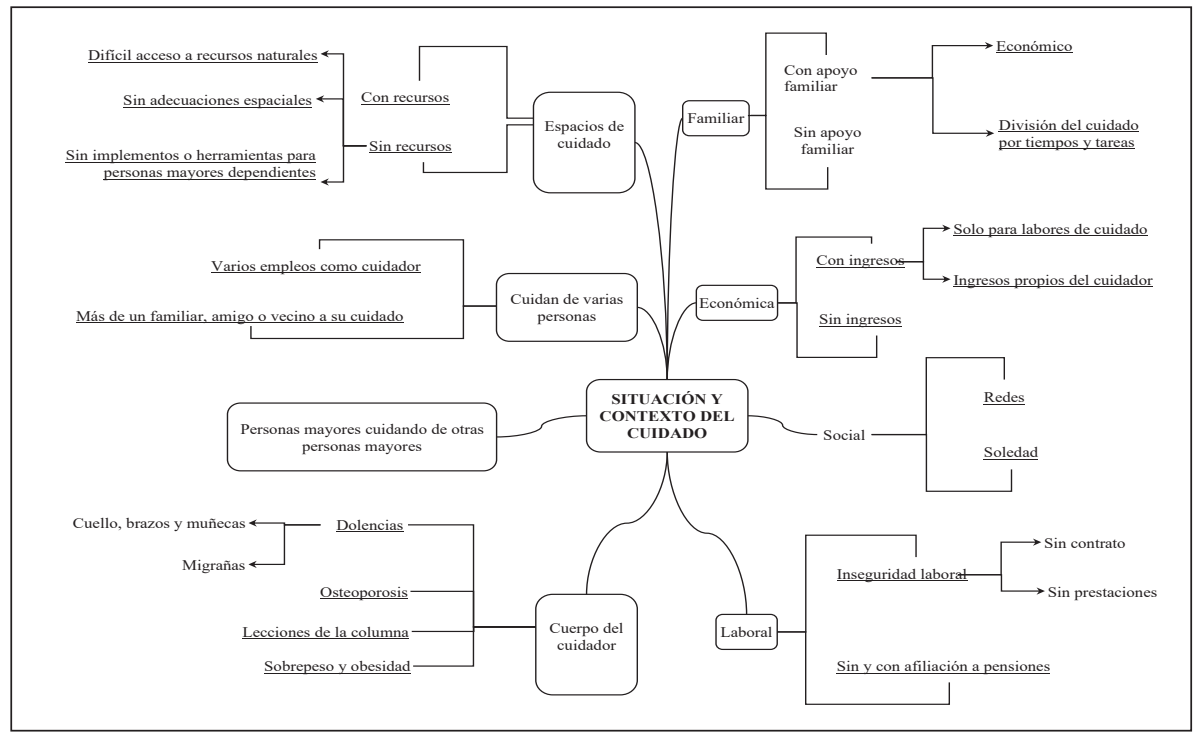

Figura 1. Situación del cuidado

Fuente: Elaboración propia

\section{Los significados del cuidado}

Dos momentos marcaron la apuesta investigativa- formativa, el inicio y el final del diplomado; al respecto se les preguntó a todos los participantes ¿qué significa para usted cuidar? Esta actividad se realizó con el pro- pósito de identificar las posibles transformaciones en los significados a partir de este proceso formativo. La figura 2 da cuenta de los significados más relevantes y cómo se transformaron.

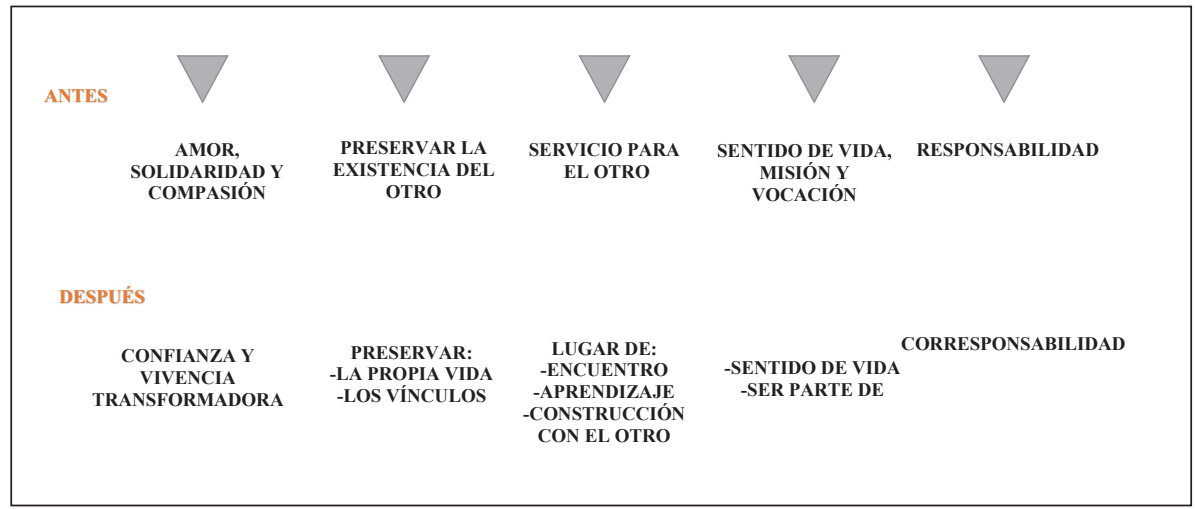

Figura 2. Transformaciones en los significados del cuidado

Fuente: Elaboración propia 
En la primera consulta, el amor como cuidado estaba representado por una entrega total hacia la persona mayor y por la expresión del cuidado como un acto de solidaridad y compasión a través del cual se manifiesta el afecto por la persona mayor. Posteriormente, este amor se trasformó en confianza y se configuró en una vivencia transformadora, rescatando la relación entre sujetos y el afecto.

La preservación de la existencia, en primera instancia, hacía alusión a preservar la salud física y emocional de la persona mayor, conservando su independencia y funcionalidad. Posteriormente se transforma en la preservación de la existencia propia, de la relación entre sujetos, de sus vínculos y del medio ambiente: "Para mi cuidar es dar lo mejor con responsabilidad y respeto, sin esperar nada a cambio. Cuidar es proteger, acompañar, estar ahi cuando lo necesiten. Cuidar, he aprendido que no solo es el otro sino yo también, es un cuidado mutuo para poder cuidar debo también cuidarme en todos los aspectos de la vida para no llegar a convertirme en un segundo paciente" [Bogotá s2-b-pe-29]

Por otro lado el servicio, inicialmente apareció como una acción hacia el otro, una disposición a ayudar y dar la mano a quien lo necesitaba; luego se convirtió en un lugar de encuentro y de aprendizaje que nutre al cuidador, a la persona a la que se cuida y a la familia. En este contexto, los cuidadores lograron reconocer que sus cuidados habían brindado un servicio a la sociedad disminuyendo la carga social del cuidado y preservando las condiciones de funcionalidad de las personas a las que cuidan, así como aportando a unas relaciones sanas.

Se debe mencionar que el sentido de vida se entendía como una misión y vocación, algo que era inherente a la persona o que, en caso de ser aprendido, requería de cualidades innatas de entrega. Posteriormente, este significado se transforma en darle sentido a la vida por el hecho de ser parte de algo; es decir, que el cuidador obtiene a partir del cuidado la posibilidad de construir nuevos vínculos, proyectos y redes de cuidado.

Otro rasgo a tener en cuenta es la responsabilidad que se convirtió en corresponsabilidad, asumiendo el autocuidado como una práctica necesaria para cuidar y concientizarse del propio proceso de envejecimiento. Por otra parte, esta corresponsabilidad evidencia el proceso de empoderamiento de la función del cuidador y de la necesidad del trabajo conjunto entre cuidador, persona cuidada, familia y sociedad.

Por último, otro de los cambios que se pueden percibir a través de la transformación en los significados del cuidado es el reconocimiento de la humanidad del cuidador y la importancia de visibilizar su labor y sus necesidades: "La evolución que ha tenido para mí la palabra cuidar, radica en reflexionar en la esencia de lo humano que posee el cuidador para dar paso a un ejercicio de cuidar. Es validar sus emociones, sus relaciones con las personas que cuida y su entorno, sus roles, los diferentes factores que intervienen en su trabajo como cuidador. En otras palabras, volverse visible como cuidador, como ser humano total, indivisible en donde todo su ser interviene en el acto de cuidar" [Bogotá $s 2-b-r-3]$.

Es importante resaltar que en todos los grupos se encontró la relación de cada significado del cuidado con la categoría transversal de relación entre sujetos, y cómo a partir de esta interacción se resignificó el cuidado incluyendo a otros actores en el cuidado, como lo son: el cuidador, el ambiente y la sociedad. Con ello se valida que "el cuidado con calidad necesariamente requiere de una interacción constante" (10). Es esa interacción la que modifica las prácticas y que, en el caso de los participantes origina nuevos proyectos para darle valor a la labor del cuidado. 


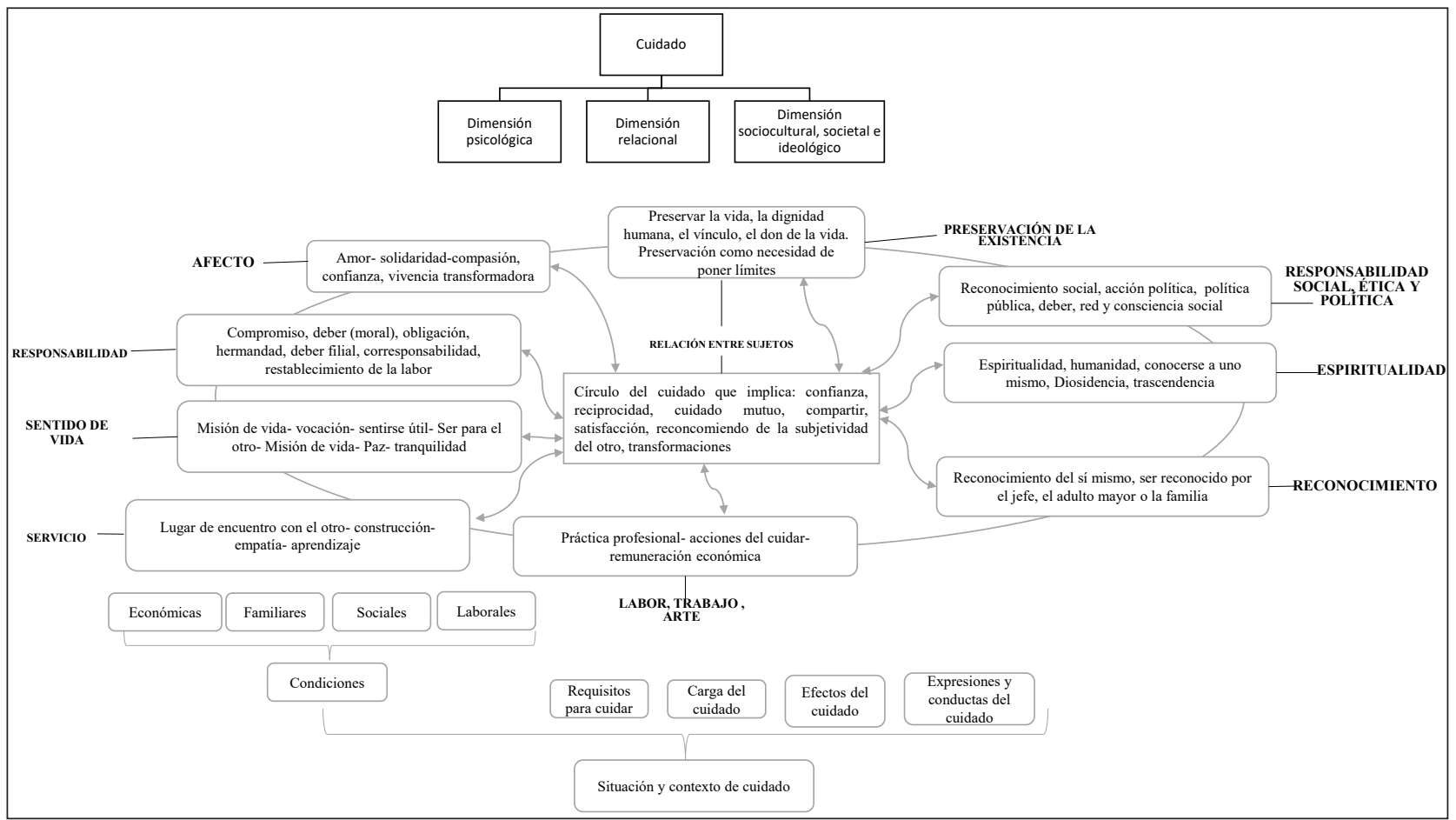

Figura 3: Interrelación del sistema categorial

Fuente: Elaboración propia

\section{Significados más representativos}

Seis significados emergieron de manera reiterativa, a saber: relación entre sujetos, preservación de la existencia, responsabilidad, servicio, sentido de la vida y amor. La relación entre sujetos implica reciprocidad, amor y compasión. Todos articulados en el sistema categorial. La preservación de la existencia es la base para desarrollar la labor de cuidado: "Requiere de un valor personal y profesional encaminado a la conservación, restablecimiento y auto cuidado de la vida que se fundamenta en la relación del paciente y cuidador, para así brindar seguridad, afecto y compromiso al paciente" [Cali s2c-rs]". Y en tanto su objetivo "es velar porque tengan mejor calidad de vida... Es devolverle la tranquilidady la dignidad al otro" [Medellín s1-m-pe 2:66].

El cuidado como responsabilidad es asumir la vida de esa persona y brindarle lo mejor de sí: "Cuidar es una gran responsabilidad con la persona que cuido y tener mucho cuidado. Darle motivación, amor, comprensión, respeto y paciencia" [Pereira s2-p-r 4:143]. De otro lado, el cuidado como servicio, implica ocuparse de las necesidades del otro, de las situaciones que la persona mayor no puede resolver por sí misma; por tanto, es actuar en beneficio de otros: "Para mí cuidar es una manera de hacer algo de provecho por la gente y para aprender muchas situaciones que hay en el proceso para llevar tranquilidad al enfermo y hacer que la persona esté bien anímicamente" [Cali s1-c-s 3:67].

El otro rasgo a tener en cuenta es el sentido de la vida que aparece ligado a una misión, es lo que se conoce como vocación. Cuidar como sentido de vida, para los participantes, tiene relación con un llamado desde el fondo de sí: "Es mi misión. Desde temprana edad he sido cuidador ya sea de mi mascota, plantas. El amor ha sido el motor. Quiero el bienestar propio y el del semejante. El sufrimiento me entristece, pero ese amor me rescata" [Pereira s1-p-sv 4:175]. Finalmente, el amor como una manifestación del cuidado, está referi- 
do a la solidaridad, a la compasión y al reconocimiento del otro: "Hoy para mí cuidar es el arte de amar al otro en todas sus formas y con todos los sentidos y no hablo del amor del corazón, hablo de la compasión, la tolerancia, la paciencia, el ponerse en el lugar del otro atendiendo a sus necesidades reales e individuales sin basarse en suposiciones" [Medellín s2-m-a 8:39].

\section{Discusión}

La presente investigación analizó los significados que construyen los cuidadores de personas mayores, en el marco de un proceso formativo sobre el cuidado. Se encontraron cambios relevantes en sus significados y en su forma de relación con las personas sujetas a su cuidado. Esto responde a que la experiencia de cuidado implica la toma de consciencia de sí mismo y del rol que se desempeña. Todo en función de las decisiones que se deben tomar a partir del reconocimiento de la situación propia, en materia de salud física, salud mental, seguridad en el ingreso para la vejez y el cuidado de sus propias redes de apoyo. En ese entorno, aparece la necesidad de crear redes de cuidado, formales o informales, de agremiarse, de conocer las normas que regulan su quehacer, de entender el contexto sociopolítico y económico en el cual desarrollan su labor, en suma, ampliar el círculo del cuidado.

Serrana (13) plantea que el significado que las personas adjudican al cuidado tiene relación con la sobrecarga y la visión totalizante del cuidado, una concepción que comprende amplios ámbitos de lo que es el cuidado (material, económico, moral y emocional). Además de las transformaciones en los significados, en el transcurso del proceso formativo se encontraron otros aspectos relevantes, que se describirán a continuación. La observación con respecto a los cuidadores, permitió entender que a medida que se avanzaba en el proceso de formación se generaba una mayor apertura a la toma de decisiones, encaminada a mejorar su propia calidad de vida y la de la persona que cuidan, algo a lo que la literatura se refiere como transformación resiliente. El cuidador en muchas ocasiones transforma de manera resiliente su forma de vida, lo que le permite un encuentro consigo mismo, dando lugar a un crecimiento espiritual y emocional, así como la comprensión del valor familiar y la solidaridad, todo esto como resultado de su encuentro en el acto de cuidar (14).

En primer lugar, se encontró que la feminización del cuidado estuvo presente en todos los grupos, así como en los testimonios y construcción con los participantes. En este sentido, es necesario profundizar en las dimensiones culturales, sociales y económicas que permitan comprender esta situación y transformarla. Los comportamientos de los cuidadores al momento de brindar cuidado están cimentados en creencias y tradiciones culturales complejas. Parece que el cuidado responde a estereotipos de género, prevaleciendo el rol en el que se ubica a las mujeres como las cuidadoras de la familia (15). La participación de los hombres en la tarea de cuidar da cuenta de posturas individuales refrendadas por discursos familiares y sociales, que resaltan el cuidado a las personas mayores como una decisión de vida, que amerita reconocimiento; sin embargo, en la mayoría de los casos, son las mujeres quienes son aceptadas culturalmente desempeñando este rol. Los casos nulos o excepcionales de hombres cuidadores en los grupos formados, muestran que no sólo han roto las representaciones frente al cuidado, sino que han demostrado compromiso y motivación por la tarea de cuidar.

De donde se infiere que, la feminización del cuidado involucra varias decisiones de carácter individual que tienen repercusión en el envejecimiento femenino. La elección de cuidar a una persona mayor generalmente implica para la mujer entre otras alternativas, dejar de trabajar o renunciar a proyectos individuales, incrementando su vulnerabilidad en los ámbitos social y de salud que podrían afectar en su propia vejez, acentuándose en gran medida la precarización en el cuidado. En el ámbito económico el cuidado genera consecuencias negativas como pensiones más reducidas, menor ahorro para afrontar la vejez y el cuidado propio hacia el futuro (17).

En segundo lugar, se evidenció también que el cuidado de los mayores en casa, generalmente se realiza sin reconocimiento económico o con un salario que garantice la seguridad económica. Al respecto, Bover y Gastaldo (18) indican que más allá de la cobertura y efectividad de los sistemas de salud, parece evidente que el cuidado de pacientes mayores y/o crónicos está mayoritariamente en manos de familiares u otros con vínculo afectivo, generalmente mujeres, que no reciben remuneración económica. El cuidado implica, por tanto, para las familias y cuidadores formales una situación compleja y cambiante, que es necesario abordar desde una perspectiva multidimensional, en donde la preparación tiene un papel clave, puesto que el cuidador que ha recibido formación sabe actuar en cada momento de la 
María Isabel Zuluaga-Callejas • María Eumelia Galeano-Marín • Caterin Bibiana Giraldo-Giraldo • Viviana Marcela Vélez-Escobar - Sandra Milena Sánchez-Vallejo • Alex Mauricio Lopera-Arango • Juliana Ortiz-Berrio • Dagoberto Barrera -Valencia

mejor manera posible; es decir, que consigue para el enfermo y para sí mismo, un clima de mayor seguridad y serenidad que le permitirá manejar, con más eficacia, la sobrecarga que produce el cuidado, a la vez que evitará muchos problemas que se pueden presentar por desconocimiento (19). Es por esto que, "en este momento vital la relación con el otro media la experiencia de salud mental, en especial el lugar que ocupa el cuidador formal, tanto en la atención de necesidades médicas como en la vinculación emocional del adulto mayor institucionalizado" (20).

Definitivamente, la situación de desprotección de los cuidadores amerita que el país defina estrategias de protección social, en clave de curso de vida. La Encuesta SABE Colombia (16), plantea cuatro aspectos negativos del cuidado: deterioro de la salud psicofísica; afectación de la situación financiera; desigualdades en salud que viven los cuidadores, como mayor morbilidad y menor participación en los programas de promoción y prevención en salud; y, por último, la alteración de las relaciones sociales del cuidador. De ahí, que sea necesario que las respuestas públicas para la atención de esta problemática se conciban como una extensión lógica del quehacer estatal, que impone ciertas obligaciones inmediatas con respecto a los que precisan ayuda y a quienes la brindan (21).

Se debe señalar que Colombia es un país con un sistema general de salud y pensiones fuertemente contributivo, ligado a la trayectoria laboral, por lo que este modelo deberá repensarse para la labor del cuidado. De acuerdo con la Política Colombiana de Envejecimiento Humano y Vejez, 2015 - 2024 (22), la protección social conlleva la integración de tres pilares básicos cuando se refiere a la protección de las personas adultas mayores y la vejez: seguridad de ingresos, atención básica de salud y organización de servicios de cuidado. En este sentido, las fuentes de provisión de protección social están agenciadas por las entidades del Estado, las organizaciones del mercado, las organizaciones sociales y comunitarias y las familias. Se debe agregar que los cuidados familiares, que se distinguen del trabajo doméstico, constituyen un campo de actuación de las políticas hacia las familias, en tanto conforman un ámbito de acción con sus actores y sus instituciones. Por tanto, la preocupación fundamental debería estar orientada en contribuir a la construcción de los cuidados como problema público objeto de políticas (23).

En tercer lugar, en el proceso de formación-investigación con cuidadores, se corrobora que las categorías cui- dador formal e informal, son insuficientes para entender las formas en las que se da el cuidado. La Organización Mundial de la Salud (24) plantea que en un extremo del espectro se encuentran los cuidadores informales que no reciben remuneración ni apoyo externo y tampoco están capacitados; estos incluyen a familiares, amigos y vecinos. En el otro extremo están los profesionales en salud altamente capacitados. En medio de ambos, hay personas que cuentan con distintos niveles de capacitación, conocimientos técnicos, estatus y remuneración. Sin embargo, la forma como se ejerce el rol de cuidador está estrechamente ligado a las circunstancias en las que se desarrolla, las cuales han sido descritas en la literatura mediante diversas tipologías del cuidado.

Por otro lado, la Encuesta SABE Colombia (16) indica que las personas cuidadoras familiares se han considerado dentro del cuidado informal, porque forman parte de la red social de la persona con dificultades o con dependencia, y en esta categoría no solo se incluyen familiares, sino amigos o vecinos; además, están fuera de la estructura formal de protección social. Tal como señalan Guzmán y Montes de Oca (25) "el cuidado familiar se encuentra enmarcado en procesos de cambios demográficos y de remodelación de los vínculos familiares". Por su parte, Rogero- García (17) plantea que el cuidado informal está formado por aquellas actividades de ayuda que van dirigidas a personas con algún grado de dependencia, que son provistas por personas de su red social y en las que no existe entidad intermediaria o relación contractual. Además, otras tipologías del cuidado se enfocan en el lugar en donde se cuida, es decir, si se cuida en una institución o en el hogar; mientras que otras hacen mayor énfasis en la existencia o no de un vínculo contractual para ejercer el rol del cuidado. No obstante, el desarrollo del diplomado permitió visualizar que dichas tipologías son complejas y en el contexto estudiado, son circunstancias que se entrecruzan, marcando dinámicas y prácticas diferentes de cuidado, caracterizadas por condiciones, riesgos y problemáticas distintos, lo que plantea la necesidad de su estudio para reconocerlas en sus particularidades.

En cuarto lugar, se pudo ratificar que cuidar tiene efectos positivos y negativos en la vida del cuidador y de quien se cuida. En la edad adulta, propone Silva (26) el cuidado puede convertirse en un instrumento de valorización de la persona mayor a partir de una doble asociación: por un lado, la asociación de la vejez con la dependencia bajo la cual se desarrollan las relaciones de cuidado; y por otro, la desvalorización sobre la 
actividad de cuidado. Ser cuidador se convierte en sinónimo de envejecer, sufrir una enfermedad o la combinación de ambas como expresión de vulnerabilidad y dependencia. Entre los efectos positivos de cuidar, se observan aquellos de carácter psicosocial, como la satisfacción de ayudar al otro y el estrechamiento positivo de relaciones (17). De la Cuesta (27) plantea que existe la necesidad de dar apoyo a los cuidadores familiares desde los conocimientos institucionales, puesto que, en el contexto de la salud y la enfermedad, es la familia la transmisora de la cultura del cuidado y del autocuidado; además de ser creadora de los discursos de salud o enfermedad proveyendo la atención y los cuidados. El cuidado conecta al que cuida con el que es cuidado. $\mathrm{Su}$ importancia reside en la presencia constante y no en la competencia técnica. De ahí, que sea una clase de relación constituida por una disposición genuina para con el otro, es la reciprocidad y el compromiso de promover el bienestar del otro (28). En otras palabras, es un trabajo de amor.

Por último, la capacitación a los cuidadores, sean formales o informales, se constituye en una herramienta para afrontar con mayor certeza su labor, pero además implica procesos de empoderamiento y reafirmación. La capacitación va más allá de los contenidos, es por eso que se "descubren" como cuidadores, toman conciencia de lo que significa cuidar, aprenden a establecer límites y a relacionarse de manera adecuada con las personas mayores que cuidan, se ponen frente a su propia vejez, se cuestionan sobre sus condiciones de trabajo, descubren la necesidad de establecer redes de cuidado y toman decisiones en diversas direcciones. Además, valoran su tarea para seguir cuidando, considerándolo como un proyecto de vida; igualmente, les sirve para descubrir que no tienen las condiciones para cuidar y así darle un cambio a sus vidas. En ese sentido, se confirma lo que plantea Chaparro (29), quien destaca que el significado del cuidado se transforma en la díada cuidador-cuidado, a partir de su experiencia. El cuidado con calidad necesariamente requiere de una interacción constante (30) y en esa medida, la capacitación transforma las prácticas de cuidado.

\section{Conclusiones}

- El cuidado implica para las familias y cuidadores una situación compleja y cambiante, que es necesario abordar desde una perspectiva multidimensio- nal, siendo la formación imprescindible. La labor que realizan los cuidadores de personas mayores, se da en contextos donde la falta de recursos económicos y de capacitación para llevar a cabo esta labor, es una constante que se observó en todos los grupos.

- En la medida que la dependencia funcional se va dando, surge la necesidad de hacer adecuaciones arquitectónicas en los hogares, que son costosas y que sólo en algunos casos, son apoyadas por fundaciones o programas estatales. Las personas mayores que actúan como cuidadoras de otras personas mayores, requieren de especial atención y apoyo, a fin de evitar que su situación de salud no se vea deteriorada por este rol.

- Hacer conscientes los significados frente a la experiencia de cuidar es lo que les ayuda a los cuidadores a moverse y pasar a la acción, en lo que respecta a cambios en sus rutinas de cuidado, adopción de prácticas de autocuidado y la implementación de estrategias para hacer frente a las dificultades familiares en torno el cuidado. Significados como: la confianza, la corresponsabilidad, la vivencia transformadora, el preservar la propia existencia a la par que se cuida al otro, surgieron en el proceso y se consolidaron como nuevas formas de asumir la labor de cuidar.

- El encuentro grupal con otros cuidadores alrededor del proceso de formación se constituye en un espacio para compartir experiencias, generar o consolidar relaciones, apoyarse mutuamente, construir redes y buscar alternativas y emprendimientos que inciden en el cuidado. Los espacios de capacitación son también escenarios para el surgimiento de liderazgos y de valoración de experiencias de vida compartidas por los participantes. Razón por la cual las experiencias que se comparten en el aula entre los cuidadores son un elemento esencial en el proceso de transformación de los significados, replanteando las motivaciones para cuidar; de igual manera, se cuestionan sobre la forma y las características del cuidado que brindan.

- Las herramientas o estrategias desarrolladas por los cuidadores frente a la carga del cuidado y los conflictos en las relaciones con las personas que rodean el cuidado, se fundamentan en poner límites y en el reconocimiento del cuidador como un ser humano. En ese sentido, el proceso de formación a través del 
María Isabel Zuluaga-Callejas • María Eumelia Galeano-Marín • Caterin Bibiana Giraldo-Giraldo • Viviana Marcela Vélez-Escobar - Sandra Milena Sánchez-Vallejo • Alex Mauricio Lopera-Arango • Juliana Ortiz-Berrio • Dagoberto Barrera -Valencia

diplomado pone en el centro del debate, la relación del cuidador con la persona que se cuida.

Como líneas de recomendación se sugiere diseñar servicios para cuidadores en el sistema de salud que minimice riesgos de envejecer en vulnerabilidad, promocionar la salud en clave de curso de vida, garantizar seguridad en el ingreso al cuidador, generar mecanismos de aseguramiento en pensiones para cuidadores. Finalmente es necesario incidir en la reglamentación del cuidado, y la protección frente a los riesgos laborales y lograr una articulación de servicios de respiro para los cuidadores.

\section{Conflicto de intereses}

Los autores declaran no tener ningún conflicto de intereses.

\begin{abstract}
$\dagger$ Este artículo es el resultado de una investigación realizada en el marco del diplomado Cuidarte, CUIDADO DEL CUIDADOR DE PERSONAS MAYORES, en las ciudades: Rionegro, Bogotá, Cali, Pereira y Medellín entre 2018 y 2020. Mediante el contrato de prestación de servicios entre PROTECCION- S. A.-Extensión de Psicología, Universidad de Antioquia. Inscrita en el Centro de Investigación de la Facultad de Ciencias Sociales y Humanas (CISH) registrada con acta 2018-23890.
\end{abstract}

\section{Referencias Bibliográficas}

1. Zuluaga M, Galeano M, Saldarriaga G. Calidad de vida en la vejez propuesta metodológica y teórica para su caracterización. Fondo Editorial FCSH Universidad de Antioquia Colombia; 2019

2. Arrubla-Sánchez DJ. Política social para el envejecimiento: el ( $\sin$ ) sentido de los argumentos. Revista Gerencia y Política de salud. 2010; 9(19): 229-242. https://doi.org/10.11144/Javeriana.rgsp9-19.pspe

3. Robledo C, Zuluaga M. Alternativas para garantizar la seguridad económica en la vejez en Colombia. Revista CES derecho. 2016; 7(1):139-150.

4. República de Colombia. Congreso de Colombia. Ley 1413 de 2010 sobre la economía del cuidado. [Internet] Bogotá D.C. Congreso de la República; 2010 [consultado el 20 de enero de 2020]. Disponible en: http://www. secretariasenado.gov.co/senado/basedoc/ley $1413 \quad 2010 . \mathrm{html}$

5. Departamento Nacional de Planeación. Bases del Plan Nacional de Desarrollo 2014-2018. [Internet]. Bogotá D. C: DNP, 2014 [consultado el 2 de enero de 2020]. Disponible en: https://colaboracion.dnp.gov.co/CDT/ PND/PND\%202014-2018\%20Tomo\%201\%20internet.pdf

6. Ministerio de Salud. Encuesta SABE Colombia 2015: de salud, bienestar y envejecimiento. [Internet]. Bogotá D.C. Minsalud, 2015 [consultado el 24 de octubre de 2018]. Disponible en: https:/www.minsalud.gov.co/ sites/rid/Lists/BibliotecaDigital/RIDE/VS/ED/GCFI/Resumen-Ejecutivo-Encuesta-SABE.pdf

7. Velásquez V, López L, López H, Cataño N, Muñoz E. Efecto de un programa educativo para cuidadores de personas ancianas: una perspectiva cultural. Rev. Salud pública. 2011; 13(4):458-469. https://doi.org/10.1590/ S0124-00642011000400006

8. De la Cuesta Benjumea C. Cuidado artesanal. La invención ante la adversidad. Medellín: Universidad de Antioquia; 2004

9. Pérez A, García L, Rodríguez E, Losada A, Porras N, Gómez M. Función familiar y salud mental del cuidador de familiares con dependencia. Aten Primaria. 2009; 41(11):621-628. https://dx.doi.org/10.1016\%2Fj. aprim.2009.03.005

10. Instituto de Mayores y Servicios Sociales. Dependencia y familia. Una perspectiva socio - económica. Colección Estudios Serie Dependencia N. ${ }^{\circ} 12013$ [Internet]. Madrid: IMSERSO; 2010 [consultado 12 marzo de 2020]. Disponible en: https://fiapam.org/wp-content/uploads/2012/10/dizy-dependencia-01.pdf

11. Roca M, Úbeda I, Fuentelsaz C, López R, Pont A, García L, Pedreny R. Impacto del hecho de cuidar en la salud de los cuidadores familiares. Atención Primaria. 2000; 26(4):217-223.

12. Estrada A, Cardona D, Segura A, Chavarriaga L, Ordóñez J, Osorio J. Calidad de vida de los adultos mayores de la ciudad de Medellín. Biomédica. 2011; 31 (4): 492- 502. https://doi.org/10.7705/biomedica.v31i4.399

13. Serrana M. Personas mayores cuidadoras: sobrecarga y dimensión afectiva. Psicología, Conocimiento y Sociedad. 2017; 7(1): 7-35. 
14. Pinto N, Barrera L, Sánchez B. Reflexiones sobre el cuidado a partir del programa "Cuidando a los cuidadores". Aquichan. 2005;5(1):128- 137.

15. Candreva A, Paladino C. Cuidado de la salud: el anclaje social de su construcción estudio cualitativo. Univ. Psychol. 2005;4(1):55-62

16. Ministerio de Salud. Encuesta SABE Colombia 2018: de salud, bienestar y envejecimiento. [Internet]. Bogotá D.C. Minsalud, 2018 [consultado el 24 de octubre de 2019]. Disponible en: https://www.minsalud.gov.co/ sites/rid/Lists/BibliotecaDigital/RIDE/DE/PS/sala-situacion-envejecimiento-2018.pdf

17. Rogero-García J. Las consecuencias del cuidado familiar sobre el cuidador: Una valoración compleja y necesaria. Index de Enfermería. 2010; 19 (1): 47-50. Disponible: http://scielo.isciii.es/scielo.php?script=sci arttext\&pid=S1132-12962010000100010

18. Bover A, Gastaldo D. La centralidad de la familia como recurso en el cuidado domiciliario: perspectivas de género y generación. Rev Bras Enferm. 2005; 58(1):9-16. Disponible: http://www.scielo.br/pdf/reben/v58n1/ a02.pdf

19. Martín Vílchez A. Necesidad de Formación en Cuidadores y Cuidadoras de personas con Demencia, [Internet]. 2014 [consultado 13 de junio de 2020]. Recuperado de: https://elnoroestedigital.com/necesidad-de-formacion-en-cuidadores-y-cuidadoras-de-personas-con-demencia/

20. González-Gaviria M, Bedoya- Gallego D. Vinculación emocional y salud mental en adultos mayores institucionalizados. Revista de psicología Universidad de Antioquia. 2019; 11(2):99-121. https://doi.org/10.17533/ udea.rp.v11n2a04

21. Comisión Económica para América Latina. Carta de San José sobre los derechos de las personas mayores de América Latina y el Caribe (CEPAL). [Internet]. San José; 2012ª . [consultado 14 de abril de 2020]. Disponible en: https://www.cepal.org/es/publicaciones/21534-carta-san-jose-derechos-personas-mayores-america-latina-caribe

22. Ministerio de Salud y Protección Social. Política colombiana de envejecimiento humano y vejez 2015-2024. [Internet]. Bogotá D. C.; 2015. [consultado 14 de febrero de 2017]. Disponible en: https://www.minsalud.gov. $\mathrm{co} / \mathrm{sites} / \mathrm{rid} /$ Lists/BibliotecaDigital/RIDE/DE/PS/Pol\%C3\%ADtica-colombiana-envejecimiento-humano-vejez-2015-2024.pdf

23. Comisión Económica para América Latina (CEPAL). Seminarios y conferencias. Políticas hacia las familias, protección e inclusión sociales. Arriagada, I. (editora). [Internet]. Santiago de Chile; 2005. [consultado 15 abril de 2020]. Disponible en: https://repositorio.cepal.org/bitstream/handle/11362/6795/1/S05683 es.pdf

24. Organización Mundial de la Salud. (OMS). Informe Mundial sobre envejecimiento y la salud. [Internet]. 2015. [consultado 12 de abril de 2016]. Disponible en: https://apps.who.int/iris/bitstream/handle/10665/186466/9789240694873 spa.pdf;jsessionid=13ADBE7BA23EBBC482DFAF8B0ACD8631?sequence $=1$

25. Guzmán J, Huenchuan S, Montes de Oca V. Redes de apoyo social de las personas mayores: Marco conceptual. Ponencia presentada en el Simposio Viejos y Viejas. Participación, Ciudadanía e Inclusión Social. 51 Congreso Internacional de Americanistas. 14-18 Julio 2003. Santiago de Chile.

26. Silva L. La relación cuidado y envejecimiento. entre la sobrevivencia y la devaluación social. Papeles de Población. 2013; 45: 49- 69

27. De la Cuesta Benjumea, C. Familia y Salud. ROL de Enfermería. 1995; 203: 21-24. https://rua.ua.es/dspace/ bitstream/10045/18356/1/Familia\%20y\%20salud.pdf

28. De la Cuesta Benjumea C. El cuidado del otro: Desafíos y posibilidades. Invest Educ Enferm. 2008; 1(25): 106-112.

29. Chaparro L. El vínculo especial del cuidado: Construcción de una teoría fundamentada. Avances en Enfermería. 2010; 28 (2): 123-133.

30. Salazar A. Conviviendo la ambivalencia en las interacciones de cuidado. Revista Cubana de enfermería. 2012; 28:(4):495-508. 